\title{
Ideal MHD Stability of Very High Beta Tokamaks.
}

\author{
M. S. Chance, S. C. Jardin, C. Kessel, \\ J. Manickam, D. Monticello \\ Princeton Plasma Physics Laboratory, Princeton, NJ 08543, USA \\ Y-K. M. Peng ${ }^{a}$ A. H. Glasser ${ }^{b}$, J. A. Holmes ${ }^{a}$, \\ D. J. Strickler ${ }^{a}$, A. Sykes ${ }^{c}$, J. C. Whitson ${ }^{a}$ \\ a Oak nidge National Laboratory, Oak Ridge, TN 37831, USA \\ ${ }^{b}$ Los Aısmos National Laboratory, Los Alamos, NM 87545. USA \\ ${ }^{\circ}$ Culham Laboratory, Abingdon, OX14 3DB, UK' \\ J. J. Ramos \\ Plasma Fusion Center, M.I.T. Cambridge, MA 02139, USA
}

\begin{abstract}
Achieving very high $\beta$ and high $\beta_{p}$ simultaneously in tokamaks generally implies that the second stability region against ballooning modes must be accessed. We describe several approaches for doing this, which are characterized by the choice of constraints imposed on the equilibrium profiles and the cross-sectional shape of the plasrna. The combination of high toroidal beta, restricting the current density to vanish at the edge of the plasma and maintaining a monotonic $q$ profile, proves to be the most stringent. Consideration of equilibria with high $\epsilon \beta_{p}$ but low $\beta$ facilitates accessibility with peaked pressure profiles and high values of $q_{0}$. Allowing the pressure gradient and, hence, the current density to be finite at the plasma edge allows all surfaces to lie within the second stability regime. For free boundary plasmas with divertors, the divertor stabilized edge region remains in the first stability regime while the plasma core reaches into the second regime. Careful tailoring of the profiles must be used to traverse the unstable barrier commonly seen near the edge of these plasmas. The CAMINO code allows us to compute $s-\alpha$ curves for general tokamak geometry. These diagrams enable us to construct equilibria whose profiles are only constrained. at worst, to be marginally stable everywhere, but do not necessarily satisfy the constraints on the current or $\beta$. There are theoretical indications that under certain conditions the external kinks possess a second region of stability at high $q_{0}$ that is analogous to that of the ballooning modes. It is found that extremely accurate numerical means must be developed and applied to confidently establish the validity of these results.
\end{abstract}

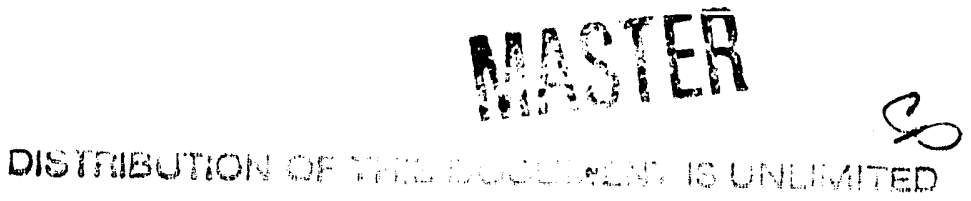




\section{INTRODUCTION}

Very high beta tokamaks with $\epsilon \beta_{p} \geq 1$ and with $\beta \gg 3 I / a B$ can substantially increase the attractiveness of a DT reactor design, while also offering the possibility of using advanced fuels. Here we report on a study to find such equilibria which are stable to all ideal MHD modes, in particular, the $n=1$ kink and the $n=\infty$ ballooning modes. These requirements lead us to consider the second region of stability. A viable configuration for a high $\beta$ second stability tokamak reactor should ideally have the following properties: $i$ ) vanishing current density at the edge of the plasma, ii) modest shaping of the cross-section, iii) monotonic $q(\psi)$ profile, and iv) stability against the external kink mode without needing a nearby conducting wall. Since much of this work is motivated by the design of the ARIES[1] device, additional constraints are imposed by engineering considerations, i.e., aspect ratio, divertor, etc.

In this paper, we show that high $\epsilon \beta_{p} \gtrsim 1$ stable configurations exist satisfying all constraints, but that these have only modest values of $\beta_{t}$. Alternatively, stable configurations are shown to exist with both $\epsilon \beta_{p} \gtrsim 1$ and with very high values of $\beta_{t}\left(\beta_{t} \gg 3 I / a B\right)$, but these require relaxing either the edge current density or the monotonic $q$ profile constraint.

The second stability equilibria develop steep gradients at the highest pressures, requiring fine meshes for numerical calculations. In fact, knowledge of the resolution needed in these studies has forced us not to rely too heavily on insights gained from our previous results on high $\beta$ studies.

The second stability studies are facilitated by the use of the CAMINO[2] code which generalizes the familiar $s-\alpha$ stability boundary $[3,4]$ to arbitrary tokamak geumetry in a three-dimensional $(s, \alpha, \psi)$ space. The normalizations of the $s-\alpha$ parameters are given by $s=2 q^{\prime} V / q V^{\prime \prime}$, and $\alpha=\left(2 p^{\prime} V^{\prime} / 4 \pi^{2}\right)\left(V / 2 \pi^{2} R_{0}\right)^{1 / 2}$, both of which $\rightarrow 0$ as $\psi \rightarrow 0$. Here, ${ }^{\prime}=\partial / \partial \psi$, and $\psi=\Psi / 2 \pi$, $\Psi$ being the poloidal flux.

An interesting set of profiles are those which are marginally stable with respect to the $s-\alpha$ stability curves at the critical point between the first and second region throughout the radius of the plasma. This technique could determine the lowest $q_{0}$ compatible with accessibility.

We have also examined the stability against the external kinks. Although theoretical considerations show that there is a second region of stability at high $q_{0}[5,7]$ which is analogous to the stability of ballooning modes, we have not yet verified this in the cases investigated, presumably because our $q_{0}$ values are still too low. The result of these studies will be described in the following sections.

\section{Access to Second Stability in the $q_{0}>1$ Regime - theoretical considerations}

The theoretical understanding of tokamak stability properties against pressure driven MHD modes is facilitated by studying the large- $q$ (both $q_{0}$ and $q_{a}$ ) regime. We consider only configurations with smooth pressure and current density profiles that vanish at the plasma edge. For these, the scaling transformation $\beta_{p} \rightarrow \beta_{p}, \beta \rightarrow \lambda^{2} \beta, q \rightarrow q / \lambda$, is an invariance of the equilibrium 
equation in the limit $q_{0}^{2} / \epsilon \gg 1$. It has been shown[5] that the ideal MHD stability equations are also invariant under such scaling for $q_{0}^{2} / \epsilon \gg 1$ and $n q \gg 1$, where $n$ is the toroidal wavenumber. In this limit, and up to corrections of the order of $1 / n q$, the stability properties of all $n$-modes become equivalent. This has some important consequences, depending on whether or not a stable access to the second stability regicn for $n=\infty$ ballooning modes exists at high $q_{0} \cdot[6, T]$

If no stable path into the second stability regime exists at high $q_{0}$, then the first stability limit must be expressed in terms of invariant parameters. The only such parameter that reflects naturally the fact that the beta limit results from a competition between the pressure gradient and the magnetic shear is $\epsilon \beta_{p} q_{0} / q_{\text {. }}$ or equivaiently $q_{0} \beta a B / I$. Therefore the Troyon form of the beta limit must be generalized to $q_{0} \beta a B / I<C_{R}\left(q_{0}\right)$ whers, in the considered case of inaccessible second stability, $C_{R}\left(q_{0}\right)$ is a weak function of $q_{0}$ that becomes independent of $q_{0}$ and $n$ as $n q_{0} \rightarrow \infty$.

If, on the other hand, a stable access to the second stability region for $n=\infty$ ballooning modes exists when $q_{0}>q_{0}^{\text {crit }}$, then all $n$-modes are stable or at worst have growth rates that decay as $1 / n q_{0}$ as $n q_{0} \rightarrow \infty$.

These theoretical results have been verified numerically with the PEST codes, for tokamak cross sectional geometries with inaccessible second stability region such as a large aspect ratio circle[5] and a small aspect ratio ellipse[i]. as well as for the cases of accessible second stability region discussed below in Sec. 3.1. This opens the possibility of considering a new tokamak operating regime with moderate 3 , high $\beta_{p}$ and relatively low current.

\section{Numerical Results}

\subsection{Maximizing Beta}

The global MHD stability properties of families of equilibria can be summarized in the space defined by $\left(q_{*} / q_{0}, q_{0}, \epsilon \beta_{p}\right)$, where $q_{*} \equiv \pi a^{2} B_{t}\left(1+\kappa^{2}\right) /\left(\mu_{0} R I_{p}\right)$. and $\epsilon \beta_{p} \equiv 8 \pi^{2}<p>a^{2} \kappa /\left(\mu_{0} I_{p}^{2}\right)$. The ratio $q_{*} / q_{0}$ represents the peakedness of the current density profile. For the configuration $A=3.0, \kappa=1.60$, and $\delta=0.4$, the diagrams are shown in Figs. 1(a) and 1(b), looking at the $q_{*} / q_{0}$ versus $\epsilon \beta_{p}$ plane. Figure 1(a) displays projections of various $q_{0}$ values showing the stability boundaries to $n=\infty$ ballooning modes. For the cases shown in this figure the current density, pressure, and pressure gradient go to zero at the plasma edge. The diagram indicates that for peaked current density profiles there exists a value of $q_{0}$ with access to a second stable region. The diagram further shows that as the current profile is broadened the value of $q_{0}$ allowing access to. or even the presence of, a second stable region increases.

In the global parameter space, we can express $\beta_{t}$ as

$$
\beta_{t}=\frac{1}{q_{0}^{2}} \frac{\epsilon \beta_{p}}{\left(q_{*} / q_{0}\right)^{2}} C(\kappa),
$$

where $C(\kappa)$ depends only on the plasma shape. A stable operating point with the largest $\beta_{t}$ would be in the lower right corner of Fig. 1(a), with $q_{0}$ as low as possible. The instabilities that occur in this region of parameter space are 
localized near the plasma edge. We will show that one approach to avoid instability in this region is to allow the pressure gradient $p^{\prime}(\psi)$ to be non-zero at the plasma edge, thus implying that the current density also must be non-zero there. Figure 1(b) illustrates how significant gains can be obtained in the maximum stable $\beta$ value by allowing finite edge gradients. The curve marked $J(a)=0$ on this diagram is the same as the $q_{0}=2$ curve in Fig. 1(a). The lower stability curve marked $J(a) \neq 0$ also has $q_{0}=2$, but allows finite edge gradients. We see from the $\beta_{t}$ contours also on this graph that the maximum stable $\beta_{t}$ value has increased from about $3 \%$ to $18 \%$ by this modification of the profiles.

By examining the local $s-\alpha$ diagrams from CAMINO at different surfaces, we see that there is a qualitative change that occurs on these diagrams for surfaces near the edge when going from low to high $\beta$ configurations as shown in Figs. $2(a)$ and $2(b)$. At low $\beta$, the equilibrium profile traces a trajectory that passes under the unstable region in the $s-\alpha$ diagram. Most of the plasma is in the second region of stability (towards the right in the diagram), while the edge of the plasma is in the first stable region (towards the left in the diagram). The connecting transition region lies in the stable zone with $s>0$ everywhere. A typical low $\beta$ stable trajectory is marked "Method I" in Fig. 2(a).

In contrast, we show the instability regions at high $\beta$ in Fig. 2(b). The instability region in the $s-\alpha$ plane for the flux surfaces near the plasma center remains relatively unchanged. However, for surfaces near the plasma edge, the instability region descends downward in the $s-\alpha$ plane, intersecting the $s=0$ axis. This behavior prevents a stable trajectory connecting the first and second stable regimes, unless the trajectory lies partially in the region where $s<0$. Thus there are no high $\beta$ staile equilibrium that simultaneously satisfy the constraints that $J(a)=0$ (implying $p^{\prime}(a)=0=\alpha$ ) with monotonically increasing $q$-profiles $(s>0)$ everywhere.

In the discussion of Fig. 1(b) we showed the advantage of allowing finite $p^{\prime}$ at the plasma edge, corresponding to "Method II" in Fig. 2(b). In Sec. 3.2 we discuss another possible stable trajectory with $s<0$ over some region ("Met hod III").

\subsection{Divertor D-Shaped Tokamaks}

High beta stability in divertor $\mathrm{D}$-shaped plasmas are of interest because of the steep pressure gradients just inside $\psi_{\mathbf{x}}$, observed in the H-mode plasmas[8]. $A$ recent theoretica! work[9] suggested that an $X$-point inboard to the plasma major radius tends to stabilize the ballooning modes in its vicinity. However, tile results in Sec. 3.1 indicate that the edge region of limiter D-shaped plasmas with vanishing edge currents often tends to remain unstable in otherwise stable plasmas of the second regime. Ideal MHD stability analysis on flux surfaces arbitrarily close to $\dot{\psi}_{x}$ is therefore needed to clarify these apparently opposing effects in very high $\beta$ equilibria. For this purpose we developed new formulae[10] to solve the ballooning coordinates $(\zeta, \beta)$ and the ballooning equation simultaneously on any closed flux surface in the $(R, Z)$ coordinates.

The ballooning stability analysis is carried out for families of FCT equilibria (see Sec. 3.3.2). An example with $A=4.5, \kappa_{x}=2.0, \delta_{x}=0.7, q_{0}=1.78, q_{95}=$ 
4.3 and $\beta_{p}=0.68-3.9$ is of particular interest. In this case, relatively broad profiles of $q(\psi)$ and $p(\psi)$ that ensure a zero edge current density are used, giving $\ell_{i}=0.65-0.47$ and $I / a B=0.70-0.93 \mathrm{MA} \mathrm{m}^{-1} \cdot \mathrm{T}^{-1}$. The results of ballooning stability for the entire divertor plasma are plotted in Fig. 3.

As can be seen, the region immediately within the divertor edge remains stable despite large increases in the pressure gradient. This confirm: the previous analytic results by Bishop [9], and is consistent with the experimental indications of the H-mode plasmas[8]. However, this region remains in the first regime since the stability degrades with an increasing pressure gradient. On the other hand, the plasma core is clearly in the second regime, as is evidenced by an improving stability with the increasing pressure gradient. There nevertheless exists a small zone of relatively unstable flux surfaces between these two regions of strong stability. instability is seen to set in when $\epsilon \beta_{p} \geq 0.6$, and migrate towards the edge as $\beta$ increased further. Stable passage to the second regime in $D$-sluped divertor plasmus with a vanishing edge current therefore must include this first-regime edge plasma, in contrast with the approach discussed above.

The results of Fig. 3 also suggest that some profile modification is needed near the edge to obtain complete ballooning stability for high values of $\epsilon \beta_{p}$. We have achieved this for divertor plasmas by decreasing the pressure gradient in this region of instability. Combining with our procedure to 'freeze' in the $q$ profile in the interior of the plasma while enforcing the condition that the current density vanish at the plasma boundary, this can be achieved by reversing the shear (causing $q^{\prime}<0$ ) in a small region near the plasma boundary. The resulting trajectories in $s-\alpha$ space are similar to those marked "Method III" in Fig. 2(b). We have verified that with these pedestal profiles, the entire plasma can remain stable to ballooning modes for $\epsilon \beta_{p} \gtrsim 1$ and for $\beta \gtrsim 10 I / a B$.

\subsection{Numerical Considerations}

\subsubsection{Fixed-Boundary Plasmas}

We have increased our capability to perform the studies presented here: A fixed boundary flux coordinate equilibrium code[11] now explicitly accepts the pressure $p(\psi)$ and the current density $J_{\mathrm{oh}}(\psi) \equiv\langle\mathbf{J} \cdot \mathbf{B}\rangle /\langle\mathbf{B} \cdot \nabla \phi\rangle$ as the two prescribed functions in the Grad-Shafranov equation, thus allowing profiles which are consistent with current drive, and in which the current density can go smoothly to zero at the plasma edge. Also, fine structures near the plasma edge have necessitated improvements in the accuracy of our numerical algorithms.

A difficulty that has plagued all of the second stability analyses is accuracy of the equilibrium, the mapping, and consequently the stability calculations. The lack of accuracy is primarily at the plasma edge, where gradients are steep for high pressure plasmas. It is typical of previous studies that equilibria used in second stability analyses have about 50 flux surfaces and 50 poloidal "angle" points $(0 \leq \Theta \leq \pi)$. This is found to be insufficient to resolve the equilibrium in high pressure plasmas. In order to examine high accuracy equilibria, a flux-coordinate fixed boundary equilibrium code, derived from [11], has been modified. The calculations can be done in $\psi$, allowing for packing of grid points towarc' the plasma edge. In addition, the ability to double the number of $\psi$ 
and $\Theta$ zones and begin the calculation from the latest converged equilibrium has been added enabling very efficient, high accuracy equilibria. A "ghost" flux surface just beyond the last plasma flux surface is introduced to allow for central differences to be used to enforce the equilibrium condition on the last surface. further improving the accuracy at the plasma edge. The mapping procedure is only used to determine metric quantities without interpolation and the stability analysis is done on the equilibrium mesh.

As an example of the required resolution for second stability plasmas, a bean shaped plasma case is examined. Shown in Fig. 4 are flux surface numbers for five different $\psi \times \Theta$ grid spacings; $51 \times 51,101 \times 101,201 \times 201,401 \times 401.601 \times 601$. Indicated on the figure are the band of flux surfaces that are high- $n$ ballooning unstable and the surface with the largest growth rate. It is clear that only after $201 \times 201$ is the unstable region in the plasma converging, i.e., the growth rate as a function of the reciprocal of the square of the number of zones shows linear convergence only after the $201 \times 201$ zone size. The actual zone size required would be a function of the gradients in the specific plasma configuration, however, the high pressure equilibria of interest for reactors require very high plasma edge resolution - better than what is typically used.

\subsubsection{Divertor Plasmas}

For divertor D-shaped plasmas, we compute free-boundary equilibria in $(R, Z)$ coordinates and exercise precise control of the plasma shape using a control matrix technique[12]. In this approach, the plasma shape is corrected before recomputation of the plasma current density $J_{\phi}$, which is used to update $\psi$ at the boundary of the rectangular mesh in $(R, Z)$. As a result, the number of updates to the free-boundary equilibrium and the total computational time are reduced by an order of magnitude from the previous approach[13]. This improvement in computational speed made it possible to solve the divertor plasma equilibrium with prescribed $q(\psi)$ and $p(\psi)$ functions, the so-called flux-conserving tokamak (FCT) equilibrium[14] in free-boundary, whilst it was successful previously only for fixed-boundary non-divertor equilibria. Numerical difficulty associated with the singularity of $q$ at the divertor flux surface $\psi_{x}$ is avoided by requiring that $F(\psi) \equiv R B_{\phi}$ be put in terms of cubic splines so as to reproduce the input $q(\psi)$ at a number of evenly distributed flux locations (up tc 8 locations), and to enforce the condition that the current density vanish at

the plasma boundary[14]. The solutions of the ballooning coordinates $(\zeta, \beta)$ and ballooning mode equation are made accurate by using bi-cubic spline fits to the equilibrium solutions of a mesh size up to $129 \times 257$ in the $(R, Z)$ coordinates.

\section{4. $n=1$ Kink Modes}

Preliminary examination of the growth rate verses beta (see Fig.5, 193 zones case) of the free boundary kink mode with the PEST code indicated that this mode might have a second region of stability like the ballooning mode. However, a convergence study of the growth rate of this mode shows that this is not so at the attempted values of $q_{0} \sim 2-3$. 
These equilibria need a large number of zones to accurately predict the growth rate of the mode because of the existence of large gradients in equilibrium quantities. The magnitude of $q^{\prime}(\psi)$ becomes very large near the plasma edge and gets larger as the $\beta$ is increased, thus making the large $\beta$ cases harder to converge. This convergence study has required running the equilibrium, mapping and stability codes with an extremely large number of zones. Cases have been run starting with a $(97,97)$ mesh in $(\psi, \theta)$ and doubling until equilibria with $\sim(769,769)$ zones are produced. We have found it important to obtain converged solutions using the same zones for the equilibrium, mapping and stability codes. Since the codes are only second order accurate, we look for the growth rate to vary linearly with the square of number of zones. Indeed for a large number of zones we find such a behavior.

A determination of the critical wall position for these modes has also been completed. For this study the critical wall position was calculated for each zone size and again a linear fit to the square of the zone size was made for each beta. The critical wall distance for complete stability to the free boundary $n=1$ mode is found to be about one-half the minor radius and increases with $\beta$.

\subsection{Marginally Accessible Profiles}

Unless the profiles chosen for the equilibrium calculations are constrained in detail the results of the parameter studies should depend on the functional forms chosen. In fact, analysis using the CAMINO code shows that the plasmas are usually either all or partially in the second region throughout their radial profile. and detailed changes in the profiles within the stable regions could quantitatively alter the stability picture. One way to make these profiles more unique is to construct them such that the plasma is marginally stable at the accessibility point for every flux surface. This point is chosen to be at the minimum of the stability boundary curve in the $s-\alpha$ plane. This procedure gives unique prescriptions for $p^{\prime}(\psi)$ and $q^{\prime}(\psi)$ and since we require $p(0)=0$ then $p(\psi)$ will be unique, but $q(\psi)$ will be determined only to within an arbitrary constant which is taken to be $q(0)$. This, as is well known, strongly influences the stability of the equilibrium. The constraints that the current or the pressure gradient vanishe at the plasma edge cannot be imposed on these CAMINO profiles. In practice we seek profiles that are only, at worst, marginally stable everywhere. In particular, since a balloon stable plasma at $\psi=0$ is generally infinitely stable there, we seek marginal stability beginning only at a finite distance away from the axis.

The results of oitaining these CAMINO profiles for two shapes - a circular, and a $\mathrm{D}$-shape with $\kappa=1.5$ and $\delta=0.3$, both with aspect ratio 3.0 are shown in Table 1. For the circular case, as $q_{0}$ is decreased, $q_{a}$ decreases sharply, thus flattening the current profile and causing the edge current to increase. The averaged beta, $\beta_{a v}$, together with the Troyon factor, $C_{T}$, increases such that $q_{0} C_{T}$ aproaches a constant in accordance with the results of Sec. 2, even though $J(a) \neq 0$ for these cases. Except for the vicinity of the origin the entire circular plasmas are all marginally stable, whereas the D-shaped configuration is marginal up to about 0.8 of the flux and stable from then on. Further iterations are needed to make the rest of the latter configuration completely marginal all 
the way to the edge. This would enhance the shear at the edge even more than that implied from the table.

\section{Conclusions}

The present study is highly relevant to advanced fuel reactor concepts based on a stable high $\beta$ tokamak equilibrium which is of interest to the ARIES studies[1]. The operating point discussed in Sec. 3 witn $\beta$ up to $18 \%, A \sim 3-4$, and $q_{0}=2$ should form the basis for such a reactor design. The accessibility paths demonstrated in this work lend further credibility to this approach.

One of the primary results of this study is that extremely fire resolution is required to obtain accurate numerical results for the equilibrium and stability of very high beta plasmas. It is especially important to resolve the boundary layer near the plasma edge if one is to obtain even a qualitative picture of the stability properties of these equilibria.

The CAMINO code has shown that, near the plasma edge, the instability region in the $(s, \alpha, \psi)$ space moves down at sufficiently high beta to intersect the $s=0$ axis. This may exclude the possibility of crossing back to $\alpha=0$ by passing under this curve, but keeping $s>0$. High beta stable plasma equilibria are still possible by staying to the high pressure side of the instability diagram everywhere, implying finite values of $p^{\prime}(\psi)$ and hence of $J$ at the plasma edge, or by allowing the shear to reverse sign in a small band near the outside surface.

Fo: lower beta equilibria that have the current density going to zero at the plasma boundary and have positive shear everywhere, we have shown that stable paths to high values of $\epsilon \beta_{p}$ near the equilibrium limit exist. The stability properties of these equilibria are well characterized in a "global $s-\alpha$ " space with $q_{*} / q_{0}$ as one axis and $\epsilon \beta_{p}$ as the other, and $q_{0}$ as the the third. Access to the equilibrium limit is gained for sufficiently large values of $q_{0}>2.5-3.0$, and for sufficiently large values of $q_{*} / q_{0}>2-3$. These equilibria, while being of modest $\beta$ value, can exceed the conventional $\beta$ limit, $\beta<3.0 I / a B$ by factors of 2 or more, and can exceed the rationalized $\beta$ limit, $\beta<3.0 I / a B q_{0}$ by factors of 6 or more.

For each value of $q_{0}$, there is a unique set of CAMINO or "gateway profiles" that form a line in the $(s, \alpha, \psi)$ space lying on the critical point of the $s-\alpha$ subspice everywhere. We have demonstrated that these profiles can be calculated, and may be of interest as they provide a unique prescription for accessing the second stability region on all surfaces simultaneously.

The $n=1$ kink mode stability of these very high beta equilibrium is exceedingly difficult to calculate accurately. There are indications thit the $n=1 \mathrm{kink}$ mode may restabilize at sufficiently high values of $\epsilon \beta_{p}$ and $q_{0}$, but limits on the numerical resolution capabilities prevent us from making definitive statements at present. 
ACKNOWLEDGMENT

This work supported by U.S. Department of Energy Contract Nos. DEAC02-76-CH0-3073, DE-AC02-78ET-51013, and DE-AC05-84OR21400.

9 


\section{References}

[1] R. Conn, et al., Thirteenth International Conference on Plasma Physics and Controlled Nuclear Fusion Research, IAEA-CN-53/H-1-4, Washington, D.C. (1990).

[2] M.S. Chance, in Theory of Fusion Plasmas, Proceedings, Varenna, Italy, 87 (1987).

[3] J.W. Connor, R.J. Hastie, and J.B. Taylor, Phys. Rev. Lett. 40, 396 (1978).

[4] J.M. Greene and M.S. Chance, Nucl. Fusion 21, 453 (1981).

[5] J.J. Ramos, Phys. Rev. A 42, 1021 (1990).

[6] A. Sykes and M.F. Turner, in Controlled Fusion and Plasma Physics, Proc. 9th European Conf., Oxford 161 (1979). R.C. Grinm, M.S. Chance, A.M.M. Todd et.al., Nucl. Fusion 25, 805 (1985). M. Gerver, J. Kesner, and J. Ramos, Phys. Fluid's 31, 2674 (1988).

[7] J.J. Ramos, MIT Report PFC/JA-90-31 (1990), to be published in PHYS. Lett. A.

[8] E.J. Strait, et al., Plasma Phys. Controlled Nucl. Fusion 1988 Vol. I, 83 (IAEA, 1989).

[9] C.M. Bishop, et al., Nucl. Fusion 24, 1579 (1984).

[10] Y-K. M. Peng, et al., "MHD Equilibrium and Ideal Stability of D-shaped Divertor H-Mode Plasmas," to be published.

[11] J. Delucia, S.C. Jardin, and A.M.M. Todd, J. Comput. Phys. 37, 183 (1980).

[12] D.J. Strickler, et al., Bull. Am. Phys. Soc. 34, 1972 (1989).

[13] D.J. Strickler, et al., "MHD Equilibrium Methods for ITER PF Coil Design and Systems Analysis," ORNL/FEDC,-88/7 (1989).

[14] R.A. Dory and Y-K. M. Peng, Nucl. Fusion 17, 21 (1977). 
Table 1. Resulis from the calculations of the marginally accessible profiles.

\begin{tabular}{||c||l|l|l||l||}
\hline \hline \multicolumn{1}{||c||}{} & \multicolumn{3}{c||}{ Circle } & Dee \\
\hline$q_{0}$ & 3.0 & 2.0 & 1.5 & 2.0 \\
\hline$q_{a}$ & 12.9 & 6.1 & 3.2 & 29.0 \\
$\beta_{a v} \%$ & 0.47 & 1.4 & 2.7 & 1.1 \\
$\beta_{I}$ & 1.2 & 0.95 & 0.66 & 2.4 \\
$C_{T}$ & 2.0 & 2.9 & 3.3 & 3.4 \\
$q_{0} C_{T}$ & 5.9 & 5.7 & 5.0 & 6.7 \\
Shift $/ a$ & 0.37 & 0.34 & 0.29 & 0.43 \\
\hline
\end{tabular}




\section{Figure Captions.}

Fig.1(a). Global parameter space showing ballooning mode stability boundaries for families of equilibria having several values of $q_{0}$ and zero edge current. Note that $C_{T}=\beta /\left(I / a B q_{0}\right)$.

Fig. 1(b). Same space with only the $q_{0}=2$ stability boundaries for families of equilibria with and without the $J(a)=0$ constraint. $\beta_{t}$ contours are also shown.

Fig. 2. $(s-\alpha-\psi)$ CAMINO-like diagrams showing the motion of the stability boundaries with increasing $\beta$ and equilibrium trajectories for (a) low $\beta$, high $\epsilon \beta_{p}$ sequence (Method I), and (b) high $\beta$, high $\epsilon \beta_{p}$ sequences with finite edge gradients (Method II), and with a negative shear region (Method III).

Fig. 3. The asymptotic coefficient of ballooning modes at large poloidal angles $(10 \times 2 \pi)$ for the family of FCT equilibria with $A=4.5, \kappa_{x}=2.0$, $\delta_{X}=0.7, q_{0}=1.78, q_{95}=4.3$, and the indicated values of $\epsilon \beta_{p}$ and $\beta_{a v}$.

Fig. 4. Computed $n=\infty$ ballooning instability regions, maximum growth rates and locations of most unstable surfaces for increasing numerical resolution.

Fig. 5. Free boundary $n=1$ kink mode growth rates (no wall) as a function of plasma $\beta$ for several numerical discretization and converged values. This equilibrium had $q_{0}=2$. 

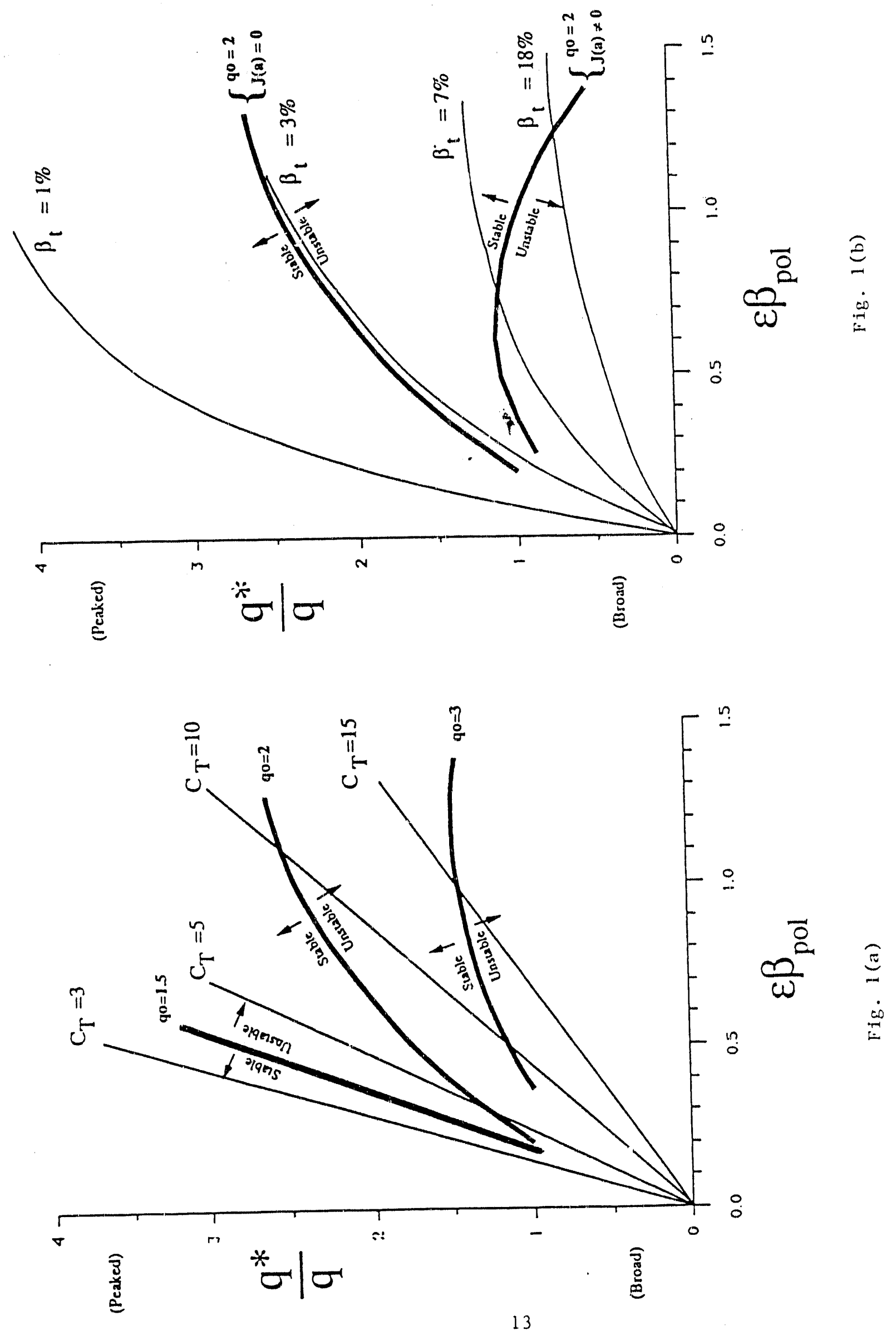


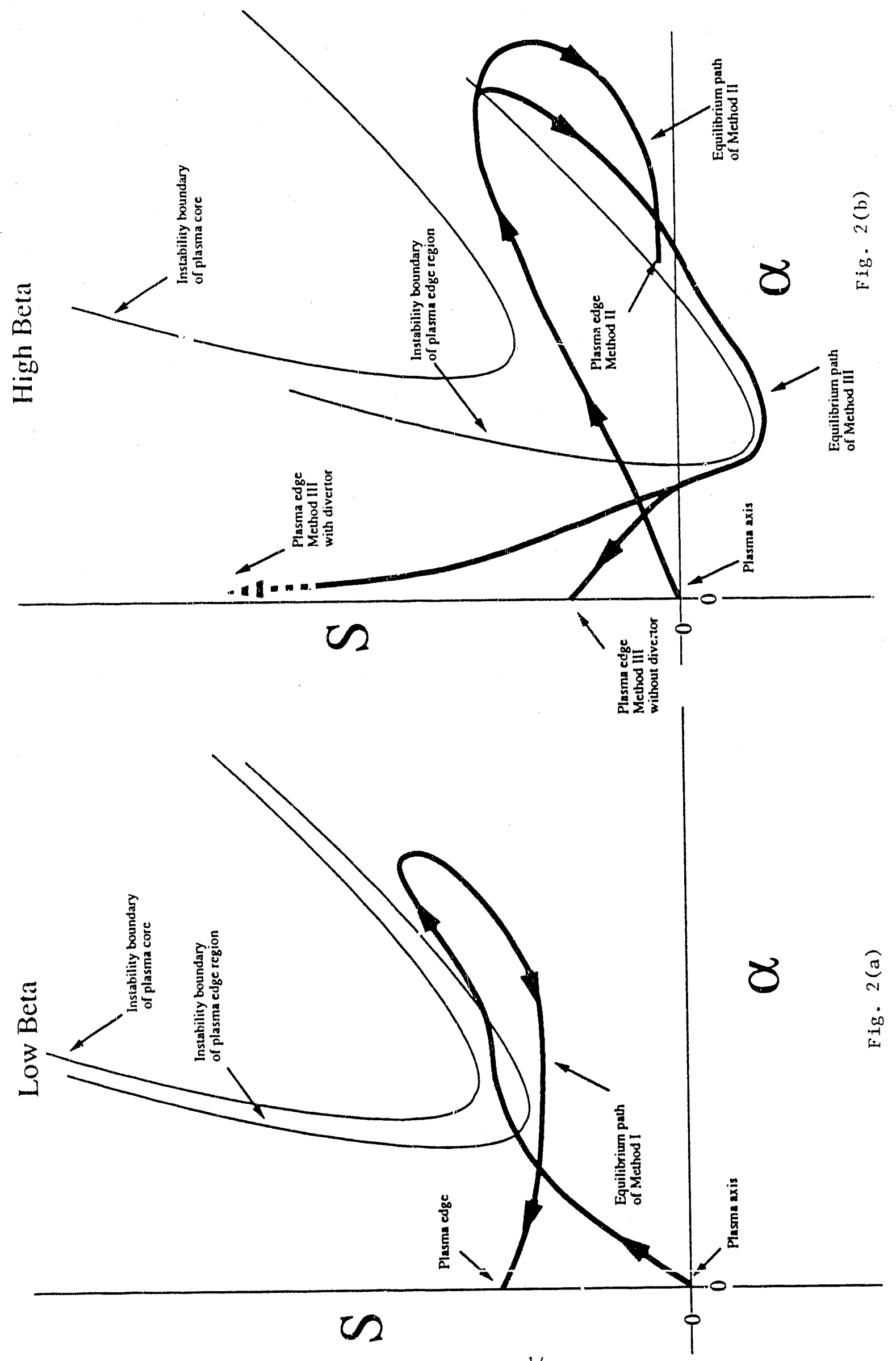




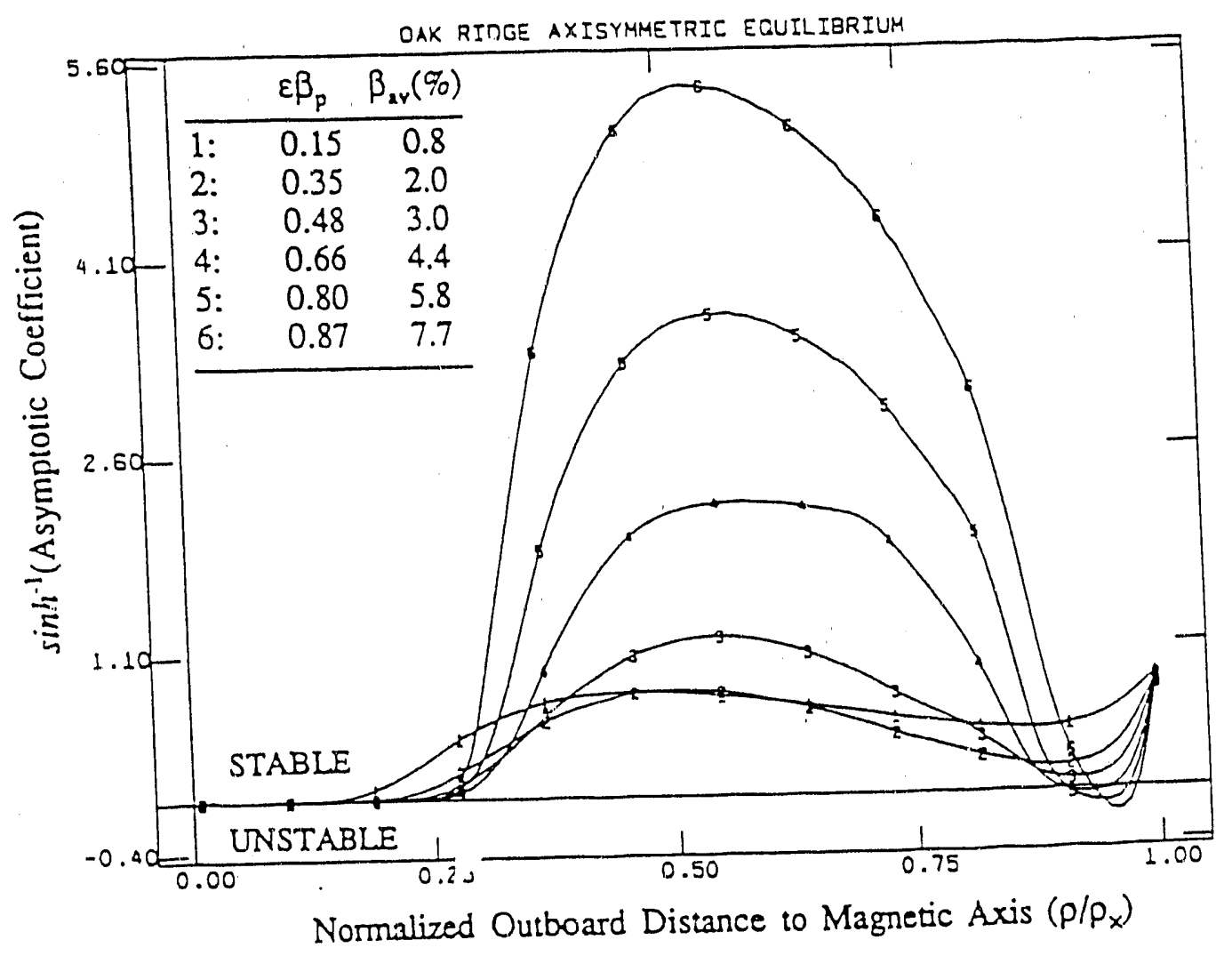

Fig. 3 

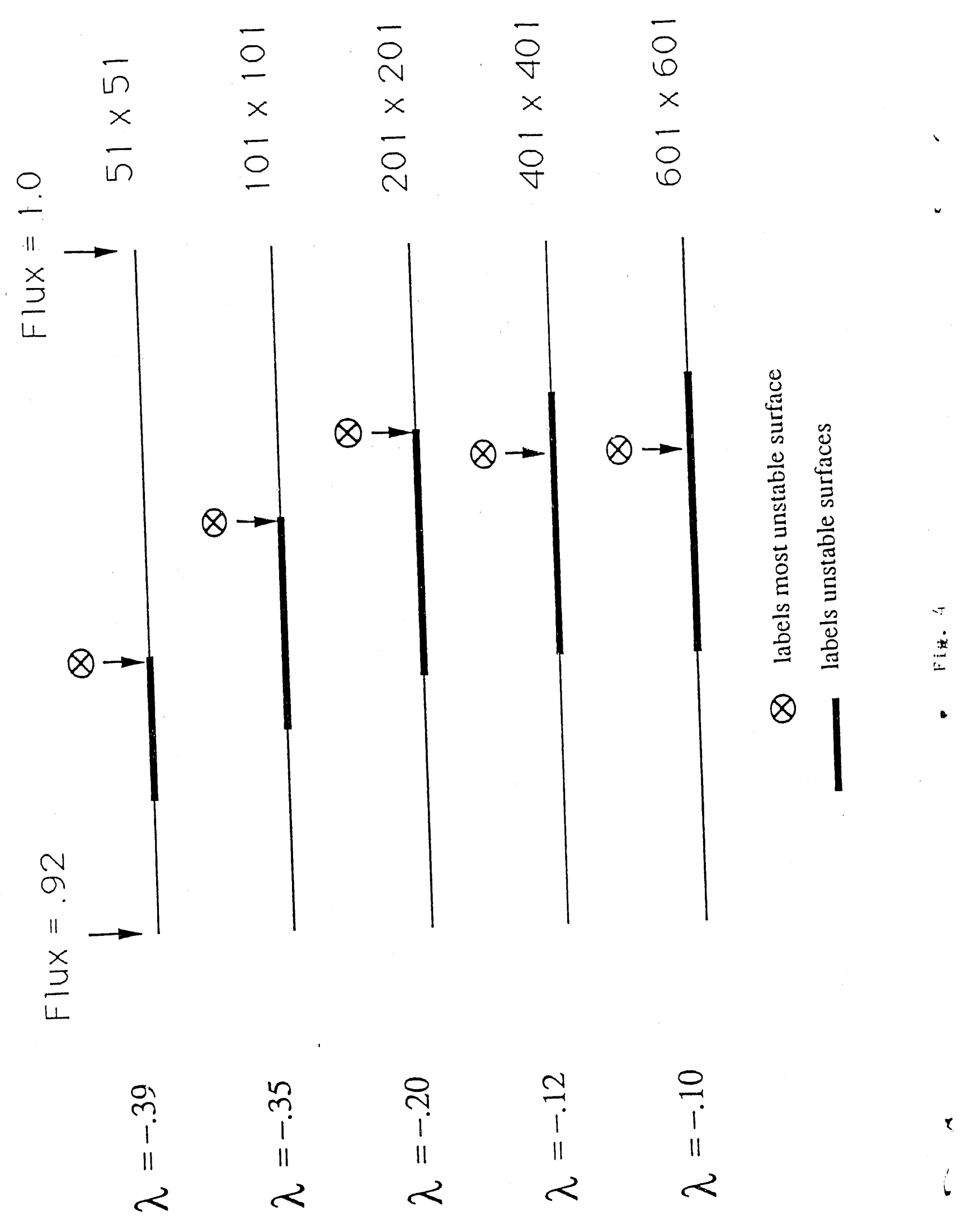


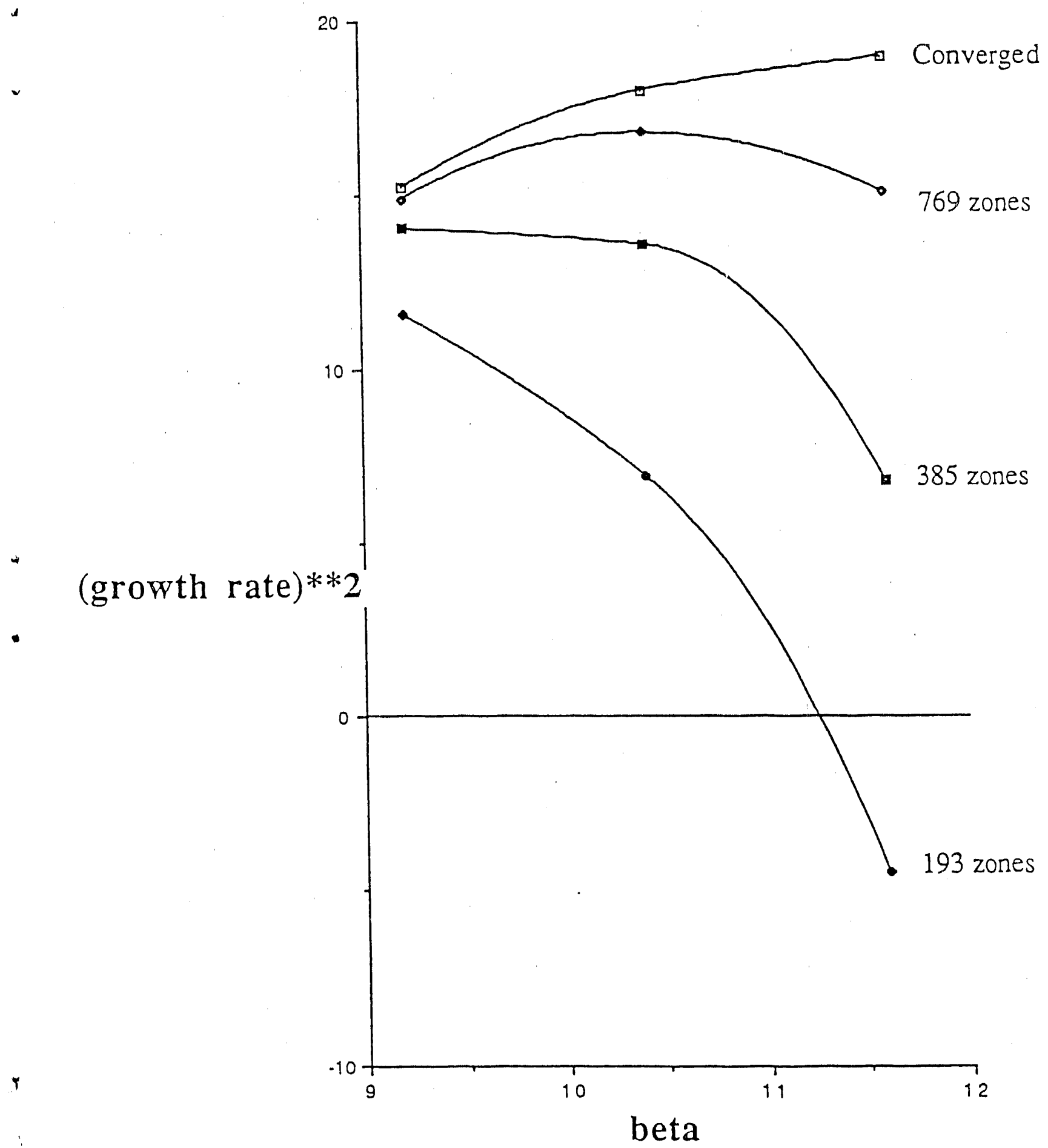

Fig. 5 

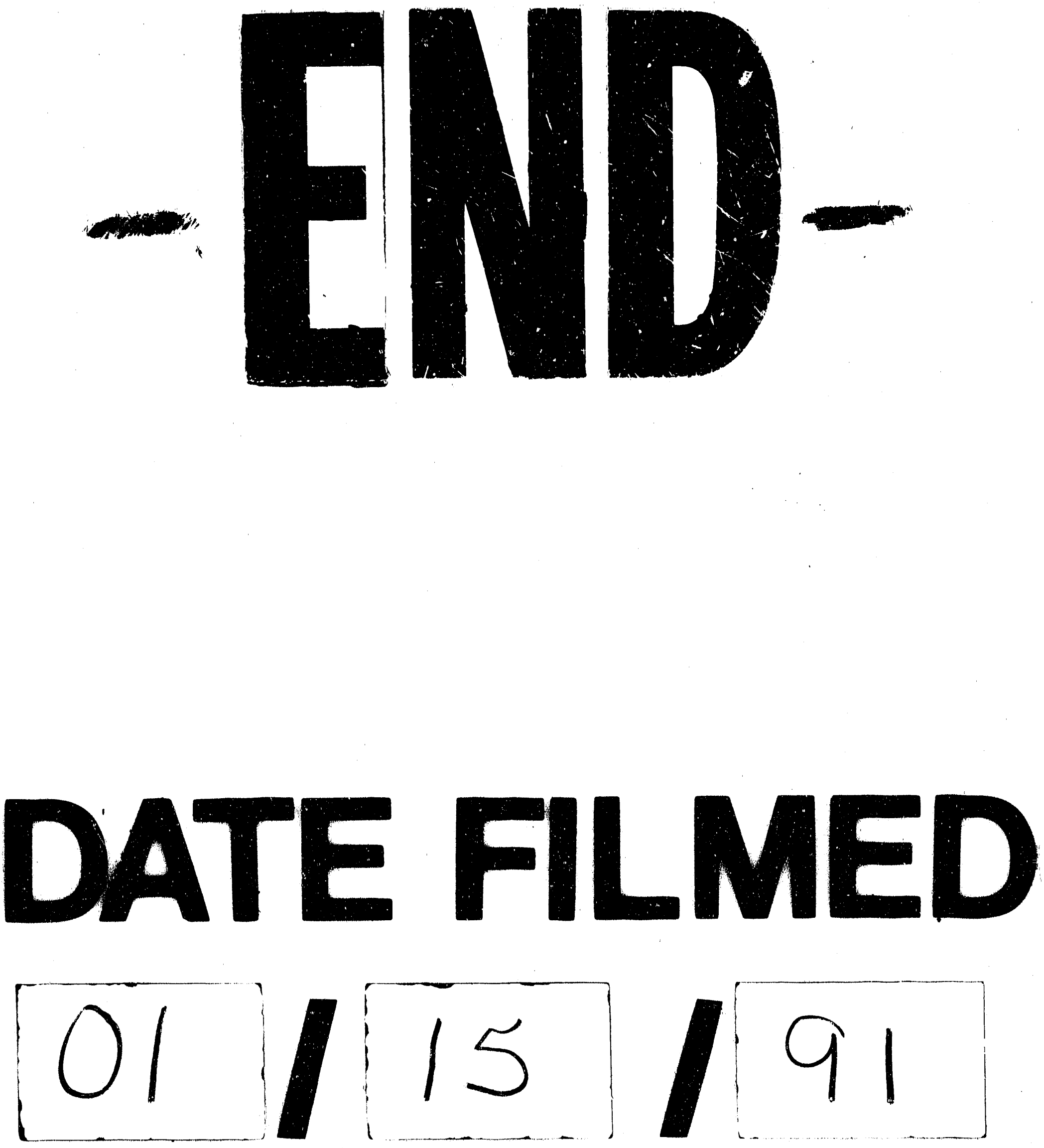
